\title{
Stem cell transplantation improves Parkinson's disease in monkeys
}

Tao, Y. et al. Nat. Med. https://doi.org/10.1038/s41591-021-01257-1 (2021)

Parkinson's disease (PD) is a neurodegenerative disorder caused by the progressive loss of dopamine (DA)-producing neurons in the substantia nigra, which results in motor symptoms such as bradykinesia, resting tremor and rigidity. Treatment with levodopa - a DA precursor - can restore DA levels in the striatum and improve motor function in patients, but its long-term use is associated with adverse effects.

Cell replacement therapy is a promising treatment option for PD. Transplantation of DA neurons or progenitors derived from pluripotent stem cells (PSCs), including induced pluripotent stem cells (iPSCs) improves motor function in animal models. However, few studies have evaluated the efficacy of autologous iPSC-based cell therapy for PD, a strategy that would eliminate the need for immunosuppression following cell transplantation.

In a new study published in Nature Medicine, a team of investigators from the University of Wisconsin-Madison evaluated the effects of autologous transplant therapy in a rhesus macaque model of PD over a two-year period. Their findings show that autologous iPSC-based treatment alleviated motor and depressive behaviors in PD monkeys, thereby confirming the clinical potential of this strategy.

The researchers induced hemiparkinsonism in ten male rhesus macaques (5-9 years old) via unilateral right intracarotid artery injection of the neurotoxin 1-methyl-4-phenyl1,2,3,6-tetrahydropyridine (MPTP). They assessed motor and behavioral functions over a 1-3-year period using a clinical rating scale (CRS) and a fine motor skill (FMS), which revealed that all monkeys developed persistent bradykinesia, postural and gait imbalances, tremors and motor skill impairments in the left hand.

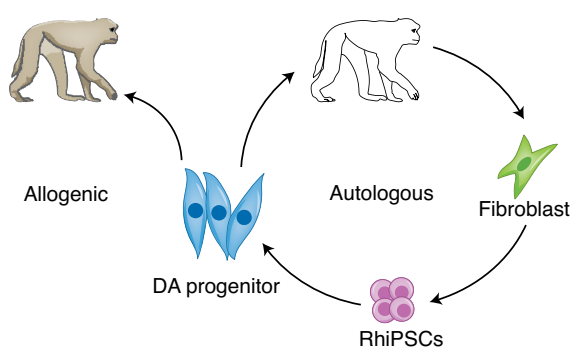

Adapted from Tao et al. (2021). Credit: Marina Spence/ Springer Nature

The MPTP monkeys were then randomly assigned to receive autologous or allogenic transplantation. Tao et al. established rhesus iPSC (RhiPSC) lines from five monkeys for autologous transplantation and used another established RhiPSC line for allogenic transplantation. The RhiPSCs were then differentiated in vitro into midbrain DA neural progenitors, labeled with GFP for tracking, and transplanted in the MPTP-treated basal ganglia of the monkeys.

CRS and FMS assessment over 24 months revealed that autologous, but not allogenic, transplantation improved motor function in monkeys. Autologous transplantation also mitigated the development of depression symptoms, whereas MPTP monkeys with allogenic grafts displayed several signs of mood disorders, including anxious pacing, lack of motivation and self-injury behavior.

Animals underwent positron emission tomography (PET) with $\left[{ }^{11} \mathrm{C}\right] \mathrm{DTBZ}$ before and after transplantation to evaluate changes in vesicular monoamine transporter type 2 (VMAT2) activity, a marker of dopaminergic sytem integrity. $\left[{ }^{11} \mathrm{C}\right] \mathrm{DTBZ}$ binding potential $\left(\mathrm{BP}_{\mathrm{ND}}\right)$ increased significantly in the striatum of autologous animals, whereas allogenic animals only showed non-significant increases in $\mathrm{BP}_{\mathrm{ND}}$.
Tyrosine hydroxylase (TH) immunostaining of brain sections revealed a higher number of DA neurons in the autologous grafts than in the allogenic grafts. $\mathrm{TH}^{+}$fibers extended a long distance away from the graft across the putamen in the autologous group, whereas few fibers grew out of grafts in the allogenic group. Immunostaining also revealed inflammatory and immunological response to allogenic grafts, which could explain the differences in the number of surviving neurons between groups.

From their data, the investigators established several correlations between putamen $\mathrm{BP}_{\mathrm{ND}}$ and motor behavioral scores (FMS, CRS and CRS recovery ratio), between putamen $\mathrm{BP}_{\mathrm{ND}}$ and the number of surviving $\mathrm{TH}^{+}$cells and between motor scores and surviving $\mathrm{TH}^{+}$cells, which suggests that PET and motor behaviors can be used to estimate the number of surviving DA neurons in future studies.

In conclusion, Tao and colleagues show that autologous and allogenic transplantations are safe in monkeys, but without immunosuppression only autologous therapy is effective.

"The need for immunosuppression and its potential complication may bring healthcare costs much higher for the allogenic strategy. In contrast, autologous cell therapy obviates the need for immunosuppression but increases the cost and wait time due to the need for iPSC generation, DA neuron differentiation and quality control. Ongoing technological innovation in the production of iPSCs and enriched DA neuron progenitors will likely make autologous transplantation therapy more effective and less costly," conclude the investigators in their report.

Alexandra Le Bras

Published online: 17 March 2021

https://doi.org/10.1038/s41684-021-00743-w 